\title{
Efecto de la adición de melatonina en el medio de maduración y/o vitrificación de ovocitos sobre la producción in vitro de embriones bovinos
}

\author{
Effect of the addition of melatonin in the oocyte maturation and/or vitrification \\ medium on in vitro production of bovine embryos
}

\author{
Maria S. Méndez ${ }^{1}$, Daniel E. Argudo ${ }^{2}$, Manuel E. Soria' ${ }^{1}$, Luis R. Galarza ${ }^{1}$, \\ Fernando P. Perea ${ }^{3,4}$
}

\section{Resumen}

Se evaluó el efecto de la adición de melatonina (Mt) en el medio de maduración y/o de vitrificación de ovocitos bovinos sobre clivaje y posterior desarrollo embrionario. Los complejos ovocito-células del cúmulo (COCs) fueron obtenidos de vacas criollas mediante la técnica de aspiración transvaginal guiada por ultrasonografía (OPU) y de ovarios de matadero (OM). Del pool de ovocitos obtenidos de ambas fuentes se seleccionaron los que tenían citoplasma homogéneo y tres o más capas compactas de células del cúmulo. Los COCs seleccionados fueron asignados aleatoriamente a cinco tratamientos: T1, madurados con Mt y vitrificados sin Mt; T2, madurados y vitrificados con Mt; T3, madurados sin Mt y vitrificados con Mt; T4 (control) madurados y vitrificados sin Mt; T5, madurados sin Mt y no vitrificados. La concentración de Mt en los medios de maduración y vitrificación fue de $0.01 \mu \mathrm{M}\left(10^{-9} \mathrm{M}\right)$. Los ovocitos fueron madurados, vitrificados, fecundados y los presuntos cigotos cultivados hasta el día 7 pos-fecundación in vitro. Los datos fueron analizados por regresión logística. Independientemente del origen de los ovocitos, el porcentaje de clivaje (PC) y de producción de embriones (PIV) fue similar entre tratamientos. El PC en los ovocitos de OPU fue mayor en T4, y la PIV fue similar entre tratamientos. En los de OM, los resultados no variaron entre tratamiento en $\mathrm{PC}$ y PIV. En conclusión, la Mt redujo el PC en ovocitos colectados por OPU, mientras que no afectó la PIV. En los colectados de OM la adición de Mt no afectó el PC ni la PIV.

Palabras clave: melatonina, maduración in vitro, vitrificación, clivaje, embriones

\footnotetext{
${ }^{1}$ Laboratorio de Biotecnología de la Reproducción Animal, Facultad de Ciencias Agropecuarias, Universidad de Cuenca, Ecuador

${ }^{2}$ Unidad Académica de Ciencias Agropecuarias, Universidad Católica de Cuenca, Ecuador

${ }^{3}$ Departamento de Ciencias Agrarias. Universidad de los Andes. Trujillo, Venezuela

${ }^{4}$ E-mail:ferromi9@gmail.com
}

Recibido: 23 de abril de 2019

Aceptado para publicación: 21 de enero de 2020

Publicado: 31 de marzo de 2020 
The effect of the addition of melatonin $(\mathrm{Mt})$ on the maturation and / or vitrification of bovine oocytes on cleavage and subsequent embryonic development was evaluated. Oocyte-cumulus cell complexes (COCs) were obtained from creole cows by ovum pick up (OPU) and by follicular aspiration from abattoir ovaries (AO). From the pool of oocytes obtained from both sources, those with homogenous cytoplasm and three or more compact layers of cluster cells were selected. The selected COCs were randomly assigned to five treatments: T1, matured with Mt and vitrified without Mt; T2, matured and vitrified with Mt; T3, matured without Mt and vitrified with Mt; T4 (control) matured and vitrified without Mt; T5, matured without Mt and not vitrified. The concentration of Mt in the maturation and vitrification media was $0.01 \mu \mathrm{M}\left(10^{-9} \mathrm{M}\right)$. The oocytes were matured, vitrified, fertilized and the presumed zygotes cultured until day 7 post-fertilization in vitro. The data were analysed by logistic regression. Regardless of the origin of the oocytes, cleavage rate (C) and embryo production (EP) was similar between treatments. The $\mathrm{C}$ in the OPU oocytes was greater in $\mathrm{T} 4$, and the $\mathrm{EP}$ was similar between treatments. In $\mathrm{AO}$, the results did not vary between treatment in $\mathrm{C}$ and $\mathrm{EP}$. In conclusion, $\mathrm{Mt}$ reduced the $\mathrm{C}$ in oocytes collected by OPU, while it did not affect EP. In the AO oocyte source, the addition of Mt did not affect the $\mathrm{C}$ nor the EP.

Keywords: melatonin, in vitro maturation, vitrification, cleavage, embryos

\section{INTRODUCCIÓN}

La vitrificación de ovocitos y embriones de mamíferos fue implementada a mediados de los años 80 (Fahy et al., 1984; Rall y Fahy, 1985) como un método alternativo a la congelación lenta. Esta biotecnología se caracteriza por la utilización de una elevada concentración de crioprotectores y el enfriamiento ultrarrápido que previene la formación de cristales de hielo dentro y fuera de la célula (De Munck y Vajta, 2017). Sin embargo, tiene el inconveniente de causar daños ultraestructurales o estructurales en los ovocitos, incluyendo cambios en la bicapa lipídica, lisis de membranas citoplasmáticas y fragmentación nuclear, así como también inducir disrupción del huso cromático, cromosomas y microfilamentos (Moussa et al., 2014; Guo et al., 2017).

La melatonina (Mt), indolamina sintetizada en la glándula pineal y en otros órganos como el ovario, es un antioxidante universal, ya que su naturaleza anfipática le permite atravesar la membrana plasmática y secuestrar directamente los radicales libres uniéndose a secuencias específicas de ADN de genes antioxidantes (Manda et al., 2007; Reiter et al., 2009), aparentemente estimulando la expresión de genes antiapoptóticos (Zhao et al., 2016). Además, interactúa con moléculas citosólicas y su mayor concentración en el líquido folicular que en el plasma incrementa el desarrollo del folículo, lo que denota un rol importante en la función ovárica (Zhao et al., 2015).

Zhao et al. (2018) demostraron que la Mt mejora significativamente la maduración citoplasmática de los ovocitos bovinos, ya que facilitó la redistribución normal de organelas, incrementó los niveles de ATP y glutatión intracelular (GSH), aumentó la expresión de genes antioxidantes y moduló los eventos relacionados con la fecundación. Además, es un agente antiapoptótico que al secuestrar las especies reactivas de oxígeno (EROs), evita la reducción del potencial de membrana 
mitocondrial impidiendo su ruptura, liberación de proteínas como citocromo c, y la activación de la cascada de enzimas caspasas que ejecutan el programa apoptótico (Zhao et al., 2016, 2018).

El bovino criollo americano, descendiente del ganado de la península Ibérica, se adaptó a las condiciones ambientales propias de la región andina de Suramérica, lo que generó biotipos caracterizados por su resistencia a enfermedades, buena eficiencia reproductiva, facilidad de parto y baja producción (Tewolde et al., 1990; Primo, 1992). En la actualidad, la población de ganado bovino criollo en Ecuador se ha reducido considerablemente, motivo por el cual las iniciativas para la preservación del germoplasma son de suma importancia para conservar este valioso patrimonio genético (Aguirre et al., 2014). Una de las formas de preservarlo es la vitrificación de ovocitos, técnica que, aunque no ha alcanzado niveles de eficacia comparables con la vitrificación de embriones, representa una alterativa viable.

Con el fin de evaluar la factibilidad de preservar el germoplasma de los bovinos criollos de los Andes ecuatorianos, se estableció como objetivo de este estudio determinar el efecto de la adición de Mt en los medios de maduración y/o vitrificación de ovocitos obtenidos mediante aspiración folicular guiada por ultrasonido y de ovarios post mortem sobre la producción in vitro de embriones bovinos.

\section{Materiales y Métodos}

\section{Productos Químicos}

Todos los productos químicos usados en este estudio fueron adquiridos en Sigma Chemical Co. (St Louis, USA), a menos que se indique lo contrario.

\section{Localización del Estudio}

Esta investigación se llevó cabo en el Laboratorio de Biotecnología de la Reproducción Animal de la Universidad de Cuenca, ubicado en la granja de Irquis, parroquia Victoria del Portete, provincia del Azuay, Ecuador, a $2648 \mathrm{msnm}$.

\section{Ovocitos de Ovarios de Matadero}

Los ovarios bovinos fueron obtenidos en el matadero municipal de Cuenca (EMURPLAG EP), y fueron transportados a una temperatura entre 35 y $37^{\circ} \mathrm{C}$ en un tiempo no mayor a tres horas desde el sacrificio al laboratorio. Los ovarios provinieron de vacas Holstein, de mestizas lecheras con alta proporción Holstein y de vacas criollas.

Los tejidos adyacentes de los ovarios fueron removidos e inmediatamente lavados con solución salina fisiológica estéril $(\mathrm{NaCl}$ al $0.9 \%$ ) a $37^{\circ} \mathrm{C}$. Los complejos cúmuloovocitos (COCs) se aspiraron de folículos de 2-8 mm de diámetro por punción directa con una aguja calibre $18 \mathrm{G}$ x 1.5 pulgadas conectada a una jeringa desechable de $12 \mathrm{ml}$. El pellet recuperado fue colocado en una placa de búsqueda para identificar y clasificar los COCs, que fueron lavados tres veces en Fluido Oviductal Sintético con Hepes (H-SOF). Solo se utilizaron ovocitos con citoplasma homogéneo y con tres o más capas compactas de células del cúmulo.

\section{Ovocitos de Vacas Criollas}

Se utilizaron 10 vacas con características de animales nativos de las alturas de los Andes ecuatorianos ( $\geq 3000 \mathrm{msnm})$, no lactantes y clínicamente sanas, las cuales fueron sometidas a repetidas aspiraciones foliculares para obtener los ovocitos. Los animales estaban alojados en la granja Irquis bajo condiciones de alimentación basada en 
pastoreo rotacional en potreros sembrados con Rye grass (Lolium perenne) y kikuyo (Pennisetum clandestinum).

Para facilitar la obtención de los COCs, las vacas fueron inmovilizadas en un brete y tranquilizadas con xilacina $2 \%(0.02 \mathrm{mg} / \mathrm{kg}$ IM), seguido del vaciamiento del contenido rectal y posterior aplicación del anestésico epidural bupivacaina $0.5 \%(0.3 \mathrm{mg} / \mathrm{kg})$. Para realizar la aspiración folicular guiada por ultrasonido (ovum pick-up - OPU), se usó el ecógrafo Aloka Prosound 2 provisto de un transductor convexo de $5 \mathrm{MHz}$ que contenía la guía de aspiración con una aguja de calibre $18 \mathrm{G} \times 1 \frac{1}{2}$ conectado a una bomba de vacío calibrada a $65 \mathrm{mmHg}$ (WTA BV 003D, Cravinhos, SP, Brasil).

Se usó un medio de recolección de ovocitos compuesto por PBS (Phosphate Buffered Saline), $1 \%$ de Suero Fetal Bovino (SFB) y 10 UI de heparina/ml. El producto de la punción (ovocitos, líquido folicular y restos celulares) fue colectado en un tubo Falcon de $50 \mathrm{ml}$ en el medio precalentado a $38.5^{\circ} \mathrm{C}$. El líquido colectado fue llevado al laboratorio, filtrado y lavado con PBS atemperado a $38.5^{\circ} \mathrm{C}$. El filtrado fue vertido en una caja de búsqueda de $95 \mathrm{~mm}$ y bajo la luz del estereoscopio (Nikon, SMZ 745T, Japón) se aislaron y clasificaron los COCs, utilizándose únicamente los que tenían citoplasma homogéneo y estaban rodeados por tres o más capas compactas de células del cúmulo.

\section{Maduración in vitro (MIV)}

Los COCs seleccionados fueron incubados en microgotas de $50 \mu 1$ en un medio de maduración TCM 199, suplementado con $10 \% \mathrm{de} \mathrm{SFB}, 100 \mu \mathrm{g} / \mathrm{ml}$ de piruvato de sodio, $0.75 \mathrm{mg} / \mathrm{ml}$ de L-glutamina, $0.01 \mathrm{UI} / \mathrm{ml} \mathrm{de}$ FSH-rh y $250 \mu \mathrm{g} / \mathrm{ml}$ de gentamicina, en una estufa (Memmert, INCO 108, Alemania) de $\mathrm{CO}_{2}$ al $5 \%$, humedad del $90 \%$ y temperatura de $38.5^{\circ} \mathrm{C}$ por 22 horas.

\section{Vitrificación y Calentamiento}

Los ovocitos fueron desprovistos parcialmente de las células del cúmulo (denudados) por pipeteo moderado y expuestos a una solución de mantenimiento (SM; H-SOF + $20 \%$ de SFB) por al menos 1 minuto. Luego se colocaron por 5 minutos en la solución de vitrificación 1 ( $\mathrm{SM}+7 \%$ de etilenglicol y $7 \%$ de dimetilsulfóxido). Inmediatamente se transfirieron a la solución de vitrificación 2 (Sucrosa $0.5 \mathrm{M}+16.5 \%$ de etilenglicol y $16.5 \%$ de dimetilsulfóxido [DMSO]) por 20 a 30 segundos, al cabo de los cuales los ovocitos fueron cargados individualmente por capilaridad, con un volumen mínimo de medio, en el dispositivo de vitrificación Cryotop (Vajta et al., 1998) y seguidamente sumergidos en nitrógeno líquido.

Los COCs se calentaron sumergiendo la punta del Cryotop en $800 \mu 1$ de $0.5 \mathrm{M}$ de sucrosa (S $0.5 \mathrm{M})$ por 2 periodos de $5 \mathrm{mi}$ nutos, luego por 5 minutos en un medio de $400 \mu 1$ de $\mathrm{S} 0.5 \mathrm{M}+400 \mu 1$ de SM y después en un medio de $800 \mu 1$ de SM durante 5 minutos. Finalmente fueron incubados durante 2 horas en TCM 199 suplementado con $20 \%$ de SFB en un ambiente con $5 \%$ de $\mathrm{CO}_{2}$, $38.5^{\circ} \mathrm{C}$ y humedad de saturación.

\section{Fecundación in vitro (FIV)}

Transcurrido el tiempo de incubación, los ovocitos fueron transferidos al medio de fecundación (FIV-SOF) suplementado con $50 \mu \mathrm{g} / \mathrm{ml}$ de heparina, y al cual se le adicionaron $1 \times 10^{6}$ espermatozoides por $\mathrm{ml}$. Estos fueron seleccionados por gradientes de Percoll (30, 60 y 90), e incubados durante 18-24 horas en un ambiente con 5\% de $\mathrm{CO}_{2}$, $38.5^{\circ} \mathrm{C}$ y humedad de saturación.

\section{Cultivo in vitro (CIV)}

Luego de la fecundación, los presuntos cigotos fueron denudados totalmente en $\mathrm{H}$ SOF y transferidos al medio de cultivo SOF- 
citrato suplementado con 5\% de SFB e incubados a $38.5^{\circ} \mathrm{C}$ por 6 días en la estufa trigas (Thermo Fisher Scientific, Heracell 150i, USA) con $5 \%$ de $\mathrm{CO}_{2}, 5 \%$ de $\mathrm{O}_{2}$ y $90 \%$ de $\mathrm{N}$, y humedad de saturación. A las 48 horas de la FIV (24 horas de la CIV) se evaluó el clivaje y a los 7 días pos-FIV la producción de embriones. Estos fueron clasificados según los criterios de la International Embryo Technology Society (IETS) (https:// www.iets.org/) y registrados según la cantidad de mórulas y blastocistos.

\section{Diseño Experimental y Análisis Estadís- tico}

Los ovocitos de ambas fuentes (OPU y $\mathrm{OM}$ ) fueron designados aleatoriamente a cinco grupos: T1, madurados con melatonina (Mt) y vitrificados sin Mt; T2, madurados y vitrificados con Mt; T3, madurados sin Mt y vitrificados con Mt; T4 (control) madurados y vitrificados $\sin \mathrm{Mt}$; T5, madurados $\sin \mathrm{Mt}$ y no vitrificados. La concentración de Mt en los medios de maduración, vitrificación $1 \mathrm{y}$ vitrificación 2 fue de 0.01 micromolar $\left(10^{-9} \mathrm{M}\right)$.

Los datos fueron procesados mediante el programa estadístico SAS (Statistical Analysis System, 2012), utilizándose la regresión logística para comparar las proporciones de clivaje y de producción de embriones entre tratamientos. Se consideraron significantes los valores de $\mathrm{p}<0.05$.

\section{Resultados y Discusión}

En el Cuadro 1 se muestra el porcentaje de clivaje (PC) y de producción in vitro de embriones (PIV) de ovocitos madurados y/o vitrificados con Mt provenientes de OPU de vacas criollas y de ovarios de matadero. El PC de T4 y T5 fue superior y estadísticamente diferente a los demás tratamientos. Además, los ovocitos vitrificados produjeron porcentajes similares de embriones e inferiores a los producidos sin vitrificación ( $\mathrm{p}<0.01$; Cuadro 1$)$.
El Cuadro 2 indica el PC y de PIV según la fuente de los ovocitos. Considerando los obtenidos por OPU, el PC varió significativamente entre los grupos T1 y T4, entre $\mathrm{T} 2$ y $\mathrm{T} 4$, y entre $\mathrm{T} 1, \mathrm{~T} 2, \mathrm{~T} 3$ y $\mathrm{T} 4$ con T5; mientras que en PIV no hubo diferencia estadística entre T1 a T4, pero si entre estos y T5. En los ovocitos colectados de OM, tanto en PC como en PIV no hubo diferencias entre $\mathrm{T} 1 \mathrm{al} \mathrm{T} 4$, pero se encontró entre estos y T5. Al comparar tratamientos similares entre las dos fuentes de ovocitos, se encontraron $\mathrm{PC}$ estadísticamente superiores en $\mathrm{T} 1 \mathrm{y}$ T2 en OM; y con relación al PIV, únicamente se observó diferencia estadística en T2, que fue superior en OM.

En general, los resultados de este estudio demostraron que la adición de melatonina en el medio de maduración y/o de vitrificación no afectó los porcentajes de clivaje y de producción de embriones, aunque fueron inferiores a los indicados en un estudio similar (Zhao et al., 2016). En dicho estudio usaron ovarios de matadero encontrando que la adición de melatonina mejoró los porcentajes de clivaje y de producción de blastocistos con relación al grupo de ovocitos vitrificados sin adición de este antioxidante; además, los porcentajes de clivaje y de blastocistos fueron mayores a los de este estudio. Aunque las condiciones experimentales fueron diferentes (grupo racial de los donantes de ovocitos, altitud de la zona) entre ambos estudios, es poco probable que hayan sido factores determinantes para estas diferencias.

Se han señalado que el uso de melatonina exógena tiene algunas limitaciones debido a sus propiedades fisicoquímicas como su baja biodisponibilidad, corta vida media, rápida absorción y metabolismo, y baja solubilidad en el agua (Lee et al., 1994; Schaffazick et al., 2006). Así, el uso de nanocápsulas de núcleo lipídico con melatonina en su interior con el fin de mejorar la estabilidad de la molécula de Mt y mitigar su aparente reducida solubilidad en agua 
Cuadro 1. Efecto de la melatonina en el medio de maduración y/o de vitrificación sobre las tasas de clivaje y de producción de embriones

\begin{tabular}{cccccc}
\hline \multirow{2}{*}{ Tratamiento } & \multirow{2}{*}{ Ovocitos $(\mathrm{n})$} & \multicolumn{2}{c}{ Clivaje } & \multicolumn{2}{c}{ Embriones al día 7 } \\
\cline { 3 - 6 } & & $\mathrm{n}$ & $\%$ & $\mathrm{n}$ & $\%$ \\
\hline T1 & 137 & 30 & $21.9^{\mathrm{a}}$ & 5 & $3.6^{\mathrm{a}}$ \\
T2 & 147 & 33 & $22.4^{\mathrm{a}}$ & 9 & $6.1^{\mathrm{a}}$ \\
T3 & 136 & 34 & $25.0^{\mathrm{a}}$ & 11 & $8.1^{\mathrm{a}}$ \\
T4 & 132 & 38 & $28.8^{\mathrm{b}}$ & 10 & $7.6^{\mathrm{a}}$ \\
T5 & 185 & 124 & $65.0^{\mathrm{c}}$ & 65 & $35.1^{\mathrm{b}}$ \\
\hline
\end{tabular}

$a, b, c$ Valores con letras distintas dentro de columnas son significativamente diferentes $(p<0.01)$

$\mathrm{T} 1$, madurados con melatonina (Mt) y vitrificados $\sin \mathrm{Mt}$; $\mathrm{T} 2$, madurados y vitrificados con Mt; $\mathrm{T}$, madurados sin Mt y vitrificados con Mt; T4 (control) madurados y vitrificados sin Mt; T5, madurados sin Mt y no vitrificados

Cuadro 2. Efecto de la melatonina en el medio de maduración y/o de vitrificación sobre las tasas de clivaje y de producción de embriones, según la fuente de ovocitos

\begin{tabular}{|c|c|c|c|c|c|}
\hline \multirow{2}{*}{ Tratamiento } & \multirow{2}{*}{$\begin{array}{l}\text { Ovocitos } \\
\text { (n) }\end{array}$} & \multicolumn{2}{|c|}{ Clivaje } & \multicolumn{2}{|c|}{ Embriones al día 7} \\
\hline & & $\mathrm{n}$ & $\%$ & $\mathrm{n}$ & $\%$ \\
\hline \multicolumn{6}{|l|}{ Ovum pick-up } \\
\hline $\mathrm{T} 1$ & 59 & 8 & $13.6^{\mathrm{a}, \mathrm{b}, \mathrm{c}, \mathrm{A}}$ & 2 & $3.4^{\mathrm{a}, \mathrm{A}}$ \\
\hline $\mathrm{T} 2$ & 64 & 7 & $10.9^{\mathrm{b}, \mathrm{c}, \mathrm{B}}$ & 0 & $0^{\mathrm{a}, \mathrm{B}}$ \\
\hline $\mathrm{T} 3$ & 62 & 12 & $19.4^{\mathrm{c}, \mathrm{d}, \mathrm{A}}$ & 4 & $6.5^{\mathrm{a}, \mathrm{A}}$ \\
\hline $\mathrm{T} 4$ & 48 & 14 & $29.2^{\mathrm{d}, \mathrm{A}}$ & 4 & $7.5^{\mathrm{a}, \mathrm{A}}$ \\
\hline $\mathrm{T} 5$ & 87 & 59 & $67.8^{\mathrm{e}, \mathrm{A}}$ & 33 & $37.9^{\mathrm{b}, \mathrm{A}}$ \\
\hline \multicolumn{6}{|l|}{ Matadero } \\
\hline $\mathrm{T} 1$ & 78 & 22 & $28.1^{\mathrm{a}, \mathrm{B}}$ & 3 & $3.8^{\mathrm{a}, \mathrm{A}}$ \\
\hline $\mathrm{T} 2$ & 84 & 26 & $31.0^{\mathrm{a}, \mathrm{C}}$ & 9 & $10.7^{\mathrm{a}, \mathrm{C}}$ \\
\hline $\mathrm{T} 3$ & 74 & 22 & $29.7^{\mathrm{a}, \mathrm{A}}$ & 7 & $9.4^{\mathrm{a}, \mathrm{A}}$ \\
\hline $\mathrm{T} 4$ & 80 & 24 & $30.0^{\mathrm{a}, \mathrm{A}}$ & 6 & $7.5^{\mathrm{a}, \mathrm{A}}$ \\
\hline $\mathrm{T} 5$ & 98 & 65 & $66.3^{\mathrm{b}, \mathrm{A}}$ & 32 & $32.6^{\mathrm{b}, \mathrm{A}}$ \\
\hline
\end{tabular}

Valores con letras distintas en la misma columna para cada fuente de ovarios son significativamente diferentes: $^{a, d} p<0.05 ;{ }^{b, d} p<0.02$; $^{a-e, b-e, ~ c-e, ~ d-e ; ~} p<0.01 ;^{a, b} p<0.01$

Valores con letras distintas en la misma columna para tratamientos similares entre cada fuente de ovarios son significativamente diferentes: ${ }^{A, B} p<0.05 ;{ }^{B, C} p<0.01$

$\mathrm{T} 1$, madurados con melatonina (Mt) y vitrificados $\sin \mathrm{Mt}$; $\mathrm{T} 2$, madurados y vitrificados con Mt; T3, madurados sin Mt y vitrificados con Mt; T4 (control) madurados y vitrificados sin Mt; T5, madurados sin Mt y no vitrificados 
fue probado en la maduración de ovocitos bovinos y la subsecuente producción de embriones (Mt encapsulada, cápsulas sin Mt, Mt administrada en una solución), sin obtener diferencias en la tasa de maduración entre grupos, pero el porcentaje de blastocistos derivados de ovocitos que fueron madurados in vitro en presencia de Mt encapsulada fue significativamente superior (Reminião et al., 2016).

La vitrificación es un método útil para almacenar ovocitos de animales por extensos periodos de tiempo; sin embargo, el desarrollo embrionario es muy bajo comparado con los embriones obtenidos in vitro de ovocitos no vitrificados (Wiesak et al., 2017). Esto pudo comprobarse en el presente estudio, donde la tasa PIV de embriones provenientes de ovocitos no vitrificados fue similar a lo reportado en estudios en los cuales no se aplicó esta técnica de criopreservación (Tian et al., 2014; Rodrigues-Cunha et al., 2016).

En otro estudio, Zhao et al. (2015), evaluó el efecto de Mt sobre los eventos apoptóticos y la producción de EROs en ovocitos bovinos provenientes de ovarios de matadero, encontrando que la adición de $10^{-9}$ $\mathrm{M}$ de $\mathrm{Mt}$ a los medios de maduración y vitrificación resultó en un 88.1 y $48.4 \%$ de clivaje y PIV, en ovocitos con Mt solo en el medio de vitrificación fue de 80.3 y $42.3 \%$ respectivamente, mientras que en el grupo sin Mt en los medios fue de 53.3 y $26.4 \%$, respectivamente. Por otro lado, en estudios donde los ovocitos fueron madurados con $\mathrm{Mt}$ sin someterlos a vitrificación, se obtuvieron valores mayores de PC y PIV a los obtenidos en los ovocitos madurados sin Mt (Tian et al., 2014; Rodrigues-Cunha et al., 2016), lo cual demuestra el efecto antioxidante de este compuesto que actúa incluso en ovocitos no sometidos a criopreservación (Zhao et al., 2018), pero que por ser madurados en un medio artificial producen mayores cantidades de radicales libres que los ovocitos que maduran en forma natural en los oviductos de las hembras de los mamíferos.
La comparación de tratamientos iguales entre las dos fuentes de ovocitos (Cuadro 2), demostró en algunos tratamientos una mayor PC en los ovocitos obtenidos de OM cuyos valores, en general, fueron superiores a los obtenidos con ovocitos colectados con OPU. En general, la calidad de los ovocitos recuperados mediante OPU es menor debido a la forma en que son obtenidos; es decir, debido a las múltiples fuerzas físicas a las que son sometidos en el recorrido entre el ovario y el tubo de colección (Bizarro et al., 2016).

\section{Conclusiones}

- En general, la adición de Mt al medio de maduración y/o de vitrificación en las condiciones experimentales de este estudio, no mejoró la tasa de clivaje de los ovocitos vitrificados y la posterior producción de embriones in vitro.

- Cuando se consideró la fuente de los ovocitos, los obtenidos con OPU y sin Mt en el medio de maduración y de vitrificación, experimentaron un porcentaje de clivaje mayor que los tratados con $\mathrm{Mt}$, mientras que esta variable fue similar entre los grupos experimentales de los obtenidos de OM.

- En ambos casos, los porcentajes de clivaje y de producción de embriones de los ovocitos vitrificados, hayan o no estado expuestos a la Mt, fue significativamente inferior a los madurados $\sin \mathrm{Mt}$ y no sometidos a vitrificación.

\section{Agradecimiento}

Los autores agradecen a la Dirección de Investigación de la Universidad de Cuenca por el financiamiento de este proyecto de investigación (DIUC-XIII-035). 


\section{Literatura Citada}

1. Aguirre L, Apolo G, Chalco L, Martínez A. 2014. Caracterización genética de la población bovina criolla de la Región Sur del Ecuador y su relación genética con otras razas bovinas. Anim Gen Res 54: 93-101. doi: 10.1017/ S2078633613000313

2. Bizarro $C$, Zandonadi F, Miguez $S$, Seneda M. 2016. Ovum pick-up. In: Seneda MM, Silva Santos KC, Rafagnin LS (eds). Biotechnology of animal reproduction. New York, USA: Nova Science. p 157-169.

3. De Munck N, Vajta G. 2017. Safety and efficiency of oocyte vitrification. Cryobiology 78: 119-127. doi: 10.1016/ j.cryobiol.2017.07.009

4. Fahy GM, MacFarane DR, Angell CA, Meryman HT. 1984. Vitrification as an approach to cryopreservation. Cryobiology 21:407-426. doi: 10.1016/ 0011-2240(84)90079-8

5. Guo XF, Yu XL, Zhang F, Wи H, Pei $X Z, L i X X, L i$ YH. 2017. Effect of liquid helium vitrification on cytoskeleton of immature cattle oocytes. Anim Rep Sci 187: 91-99. doi: 10.1016/j.anireprosci.2017.10.010

6. Lee BJ, Parrott KA, Ayres JW, Sack RL. 1994. Preliminary evaluation of transdermal delivery of melatonin in human subjects. Res Commun Mol Pathol 85: 337-346. doi: 10.1081/DDC100102199

7. Manda K, Ueno M, Anzai K. 2007. AFMK, a melatonin metabolite, attenuates X-ray-induced oxidative damage to DNA, proteins and lipids in mice. J Pineal Res 42: 386-393. doi: 10.1111/j.1600-079X.2007.00432.x

8. Moussa M, Shu J, Zhang X, Zeng F. 2014. Cryopreservation of mammalian oocytes and embryos: current problems and future perspectives. Sci China Life Sci 57: 903-914. doi: 10.1007/s11427014-4689-z
9. Primo AT. 1992. El ganado bovino ibérico en las Américas: 500 años después. Arch Zootec 41: 421-432.

10. Rall WF, Fahy GM. 1985. Ice-free cryopreservation of mouse embryos at $196{ }^{\circ} \mathrm{C}$ by vitrification. Nature $313: 573-$ 575. doi: 10.1038/313573a0

11. Reiter RJ, Tan DX, Manchester LC, Paredes SD, Mayo JC, Sainz RM. 2009. Melatonin and reproduction revisited. Biol Reprod 81: 445-456. doi: 10.1095/biolreprod.108.075655.

12. Remião MH, Lucas CG, Domingues $W B$, Silveira $T$, Barther NN, Komninou ER, Basso AC, et al. 2016. Melatonin delivery by nanocapsules during in vitro bovine oocyte maturation decreased the reactive oxygen species of oocytes and embryos. Reprod Toxico 63: 70-81. doi: 10.1016/j.reprotox.2016.05.016

13. Rodrigues-Cunha MC, Mesquita LG, Bressan F, Collado MD, Balieiro JC, Schwarz KR, de Castro FC, et al. 2016. Effects of melatonin during IVM in defined medium on oocyte meiosis, oxidative stress, and subsequent embryo development. Theriogenology 86: 16851694. doi: 10.1016/j.theriogenology.2016.05.026

14. Schaffazick SR, Pohlmann AR, Mezzalira G, Guterres SS. 2006. Development of nanocapsule suspensions and nanocapsule spray-dried powders containing melatonin. J Braz Chem Soc 17: 562-569.

15. Tewolde A, Salgado D, Campos M, Mujica F. 1990. El papel de los recursos genéticos criollos en sistemas de producción bovina en el trópico. En: Tewolde A, Salgado D, Campos M, Mujica F (eds). Memorias Conferencia Internacional sobre Sistemas y Estrategias de Mejoramiento Bovino en el Trópico. Turrialba, Costa Rica

16. Tian X, Wang F, He C, Zhang L, Tan D, Reiter RJ, Xu J, Ji P, Liu G. 2014. Beneficial effects of melatonin on bovine 
oocytes maturation: a mechanistic approach. J Pineal Res 57: 239-247. doi: 10.1111/jpi.12163

17. Vajta G, Holm P, Kuwayama M, Booth PJ, Jacobsen H, Greve T, Callesen $\mathrm{H}$. 1998. Open Pulled Straw (OPS) vitrification: a new way to reduce cryoinjuries of bovine ova and embryos. Mol Reprod Dev 51: 53-58. doi: 10.1002/ (SICI)1098-2795(199809)51:1<53::AIDMRD6>3.0.CO;2-V

18. Wiesak T, Wasielak M, Zlotkowska A, Milewski R. 2017. Effect of vitrification on the zona pellucida hardening and follistatin and cathepsin B genes expression and developmental competence of in vitro matured bovine oocytes. Cryobiology 76: 18-23. doi: 10.1016/ j.cryobiol.2017.05.001.
19. Zhao XM, Min JT, Du WH, Hao HS, Liu Y, Qin T, Wang D, Zhu HB. 2015. Melatonin enhances the in vitro maturation and developmental potential of bovine oocytes denuded of the cumulus oophorus. Zygote 23: 52555236. doi: 10.1017/S0967199414000161

20. Zhao XM, Hao HS, Du WH, Zhao SJ, Wang HY, Wang N, Wang D, et al. 2016. Melatonin inhibits apoptosis and improves the developmental potential of vitriûed bovine oocytes. J Pineal Res 50: 132-141. doi: 10.1111/jpi.12290

21. Zhao XM, Wang N, Hao HS, Li CY, Zhao YH, Yan CL, Wang HY, et al. 2018. Melatonin improves the fertilization capacity and developmental ability of bovine oocytes by regulating cytoplasmic maturation events. J Pineal Res 64: e12445. doi: 10.1111/jpi.12445 